\title{
Thrombus Formation Processes are dependent on Endothelial Injuries: Examined by In vivo Two-photon Molecular Imaging and Laser Manipulation
}

\author{
Satoshi Nishimura ${ }^{1,2,3}$
}

IDepartment of Cardiovascular Medicine, the University of Tokyo, Hongo 7-3-1, Bunkyo-ku, Tokyo, Japan

2Translational Systems Biology and Medicine Initiative, the University of Tokyo, Hongo 7-3-1, Bunkyo-ku, Tokyo, Japan

4Jichi Medical University, Tochigi, Japan

Aim: The cellular mechanisms of thrombotic diseases associated with cardiovascular events remains unclear, largely because of an inability to visualize thrombus formation. Thus, we developed in vivo imaging technique based on single- and multi-photon microscopy and laser injuries technique to revealed the multicellular processes during thrombus development. Methods: We visualized the cell dynamics including single platelet behavior, and assessed dynamic cellular interplay in two thrombosis models using two photon microscopy to CAG-eGFP mice in which GFP was expressed ubiquitously. Hoechst was injected to visualize nucleus, and dextran to blood flow. Mice with anestesia is set on to inverted microscopy, and visualization was performed through small incision. (Figure a, b).

Results: First, we visualized that rapidly developing thrombi composed of discoid platelets without EC disruption was triggered by ROS photochemically induced by moderate power laser irradiation (Figure c). In this model, thrombus consisted by discoid platelet aggregations without leukocyte recruitment. The second model is, thrombus with EC disruption. High power laser induced EC erosion and extravasations of circulating leukocytes with thrombus development. Inflammatory cytokine, adhesion molecules dynamically control these two processes. (Figure d) As for the thrombus formation with EC disruption, chemokine expressions in endothelium and leukocyte (especially neutrophils) recruitment played a significant role in these processes. Leukocyte was immediately recruited into the subendothelial layers with bleeding and hemostatic reactions. TLR4 signaling also contributed to these steps, and pretreatmet of LPS markedly enhanced these steps. Thrombus included calcium activated cores and deformed platelets. Immigrated leukocyte also showed the increase of intracellular calcium.

Summary: These results indicated that endothelial function, especially inflammatory status, determined the thrombotic reaction. Leukocyte also contributed with TLR4 signaling. In sum, using our imaging system can be a powerful tool to analyze thrombus formation and evaluate the therapeutic strategies.

[1] Nishimura et al, 2012 Blood.

[2] Nishimura et al, $2010 \mathrm{JCI}$.

[3] Nishimura et al, 2009 Nat Med.

[4] Nishimura et al, 2013 Cell Metabolism

[5] Nishimura et al, 2008 JCI. 

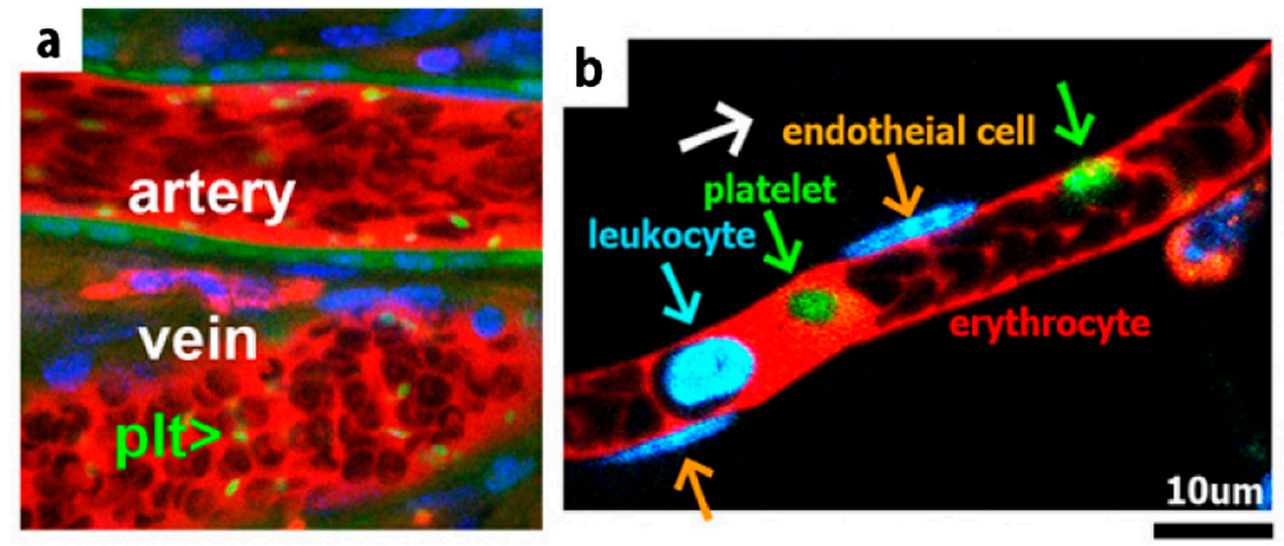

Cellular kinetics in vessels visualized by in vivo imaging

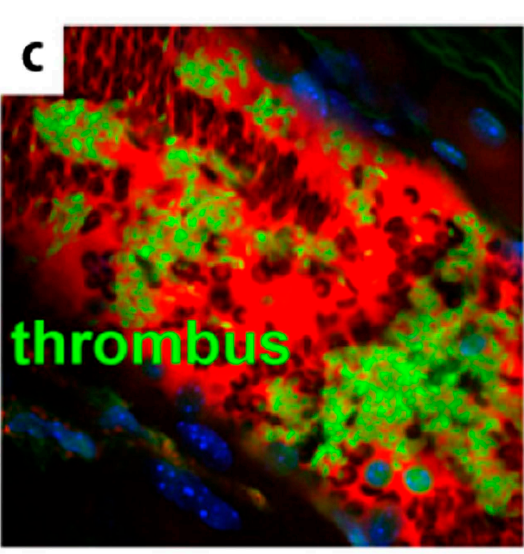

Thrombus formation without endothelium disruption

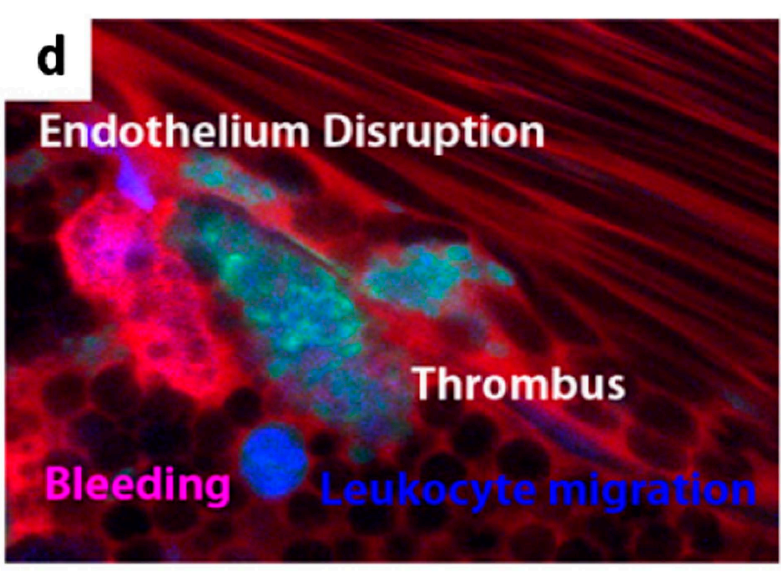

Thrombus formation with endothelium disruption

Figure: In vivo imaging of thrombus formation (Green : CAG-eGFP, Red: Dextran, Blue: Hoechst) 